Faculdade

de Ciências Econômicas UFRGS

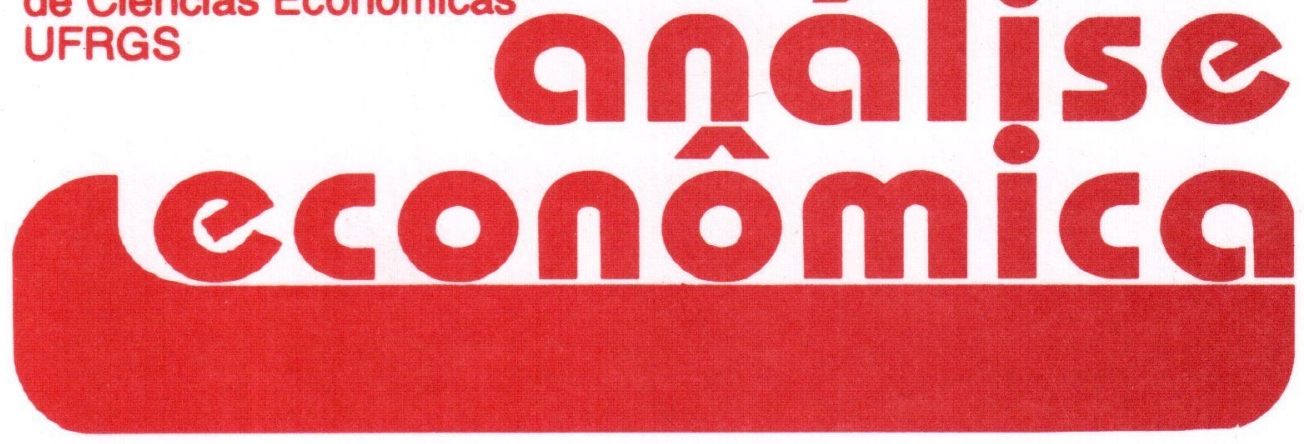

- Finance and Growth:

is Schumpeter Right?

- Philip Arestis

- Panicos Demetriades

- Repensando a

Revolução Marginalista

- Ricardo Luis C. Feijó

- O Papel dos

Rendimentos Crescentes

na Localização Econômica

- Paulo Augusto P. de Britto

- Credibilidade e Mercado Secundário da Dívida Externa Brasileira

- Paulo Calderon

- Rosa Fontes

- Fronteira de Eficiência sob Condições de Risco

- Luís A. de Araújo

- José Vicente Caixeta Filho

- A Criação de

Municípios e seu Impacto na

Qualificação do Espaço Urbano

- Maria Conceição B. Scussel

- Um Estudo Crítico das Relações entre as Poupanças Privada,

Pública e Nacional

- Martin R. Cavalcanti

- Joanílio R. Teixeira
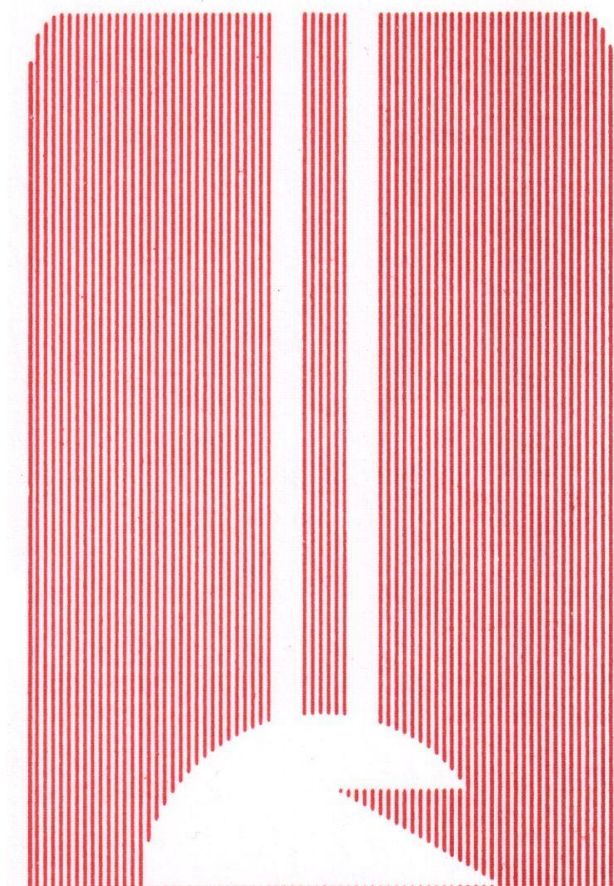

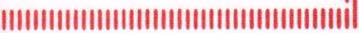

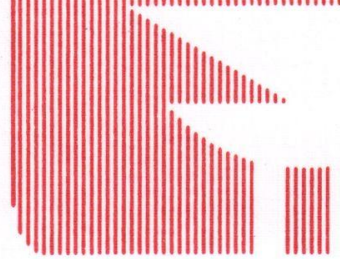

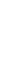


UNIVERSIDADE FEDERAL DO RIO GRANDE DO SUL

Reitora: Prof ${ }^{\mathrm{a}}$. Wrana Maria Panizzi

FACULDADE DE CIÊNCIAS ECONÔMICAS

Diretora: Prof ${ }^{\mathrm{a}}$. Otilia Beatriz Kroeff Carrion

CENTRO DE ESTUDOS E PESQUISAS ECONÔMICAS

Diretor. Prof. Fernando Ferrari Filho

DEPARTAMENTO DE CIÊNCIAS ECONÔMICAS

Chefe: Prof. Luiz Alberto Oliveira Ribeiro de Miranda

CURSO DE PÓS-GRADUAÇÃO EM ECONOMIA

Coordenador. Prof. Marcelo Savino Portugal

CURSO DE PÓS-GRADUAÇÃO EM ECONOMIA RURAL

Coordenador. Prof. Carlos Guilherme A. Mielitz Netto

CONSELHO EDITORIAL: Achyles B. Costa, Aray M. Feldens, Carlos A. Crusius, Carlos G. A. Mielitz Netto, Eduardo A. Maldonado Filho, Eduardo P. Ribeiro, Eugênio Lagemann, Fernando Ferrari Filho, Gentil Corazza, Marcelo S. Portugal, Nali J. Souza, Otília B. K. Carrion, Paulo A. Spohr, Paulo D. Waquil, Pedro C. D. Fonseca, Roberto C. Moraes, Ronald Otto Hillbrecht, Stefano Florissi, Eleutério F. S. Prado (USP), Fernando H. Barbosa (FGV/RJ), Gustavo Franco (PUC/RJ), João R. Sanson (UFSC), Joaquim P. Andrade (UnB), Juan H. Moldau (USP), Paul Davidson (Univ. of Tennessee), Werner Baer (Univ. of Illinois).

COMISSÃO EDITORIAL: Fernando Ferrari Filho, Gentil Corazza, Paulo Dabdab Waquil, Marcelo Savino Portugal, Roberto Camps Moraes.

EDITOR: Eduardo Augusto Maldonado Filho

EDITOR ADJUNTO: Gentil Corazza

SECRETARIA: Revisão de textos: Vanete Ricacheski.

FUNDADOR: Prof. Antônio Carlos Santos Rosa

Os materiais publicados na revista Análise Econômica são da exclusiva responsabilidade dos autores. É permitida a reprodução total ou parcial dos trabalhos, desde que seja citada a fonte. Aceita-se permuta com revistas congêneres. Aceitam-se, também, livros para divulgação, elaboração de resenhas e recensões. Toda correspondência, material para publicação (vide normas na terceira capa), assinaturas e permutas devem ser dirigidos ao seguinte destinatário:

PROF. EDUARDO AUGUSTO MALDONADO FILHO

Revista Análise Econômica - Av. João Pessoa, 52 CEP 90040-000 PORTO ALEGRE - RS, BRASIL

Telefones: (051) 316-3324 e 316-3440 - Fax: (051) 316-3990 rae@vortex.ufrgs.br 


\title{
FRONTEIRA DE EFICIÊNCIA ECONÔMICA SOB CONDIÇÕES DE RISCO PARA EMPRESAS AGRÍCOLAS DO SUL DE SANTA CATARINA
}

\author{
Luis Augusto Araújo* \\ José Vicente Caixeta Filho**
}

\begin{abstract}
RESUMO
O principal objetivo deste estudo é a análise da eficiência econômica de empresas agrícolas sob condições de risco, através de um modelo de fronteira, usando dados de cross-section do Sul do Estado de Santa Catarina. Identificam-se, também, ao longo da fronteira de eficiência, os pontos de máxima margem bruta e utilidade. O principal resultado da análise de eficiência é a existência de um potencial médio de crescimento da margem bruta de $49,9 \%$ e de $62,6 \%$, quando o produtor adotar, respectivamente, o plano de máxima utilidade e de máximo retorno, dada a disponibilidade de fatores de produção, os padrões técnicos e econômicos das alternativas de produção e o nivel de risco que deseja suportar.
\end{abstract}

Cód. AEA: 716

Palavras-chave: Fronteira de eficiência econômica, risco, programação linear.

\section{ABSTRACT}

The main objective of this study is to analyze the economic efficiency of farms under risk conditions, through a frontier model using cross section data from the Southern region of the State of Santa Catarina. Specific objetives include the identification of maximum Gross Margin and utility points along the efficiency frontier. The results show that there is a medium potential for the gross margin to increase. Gross Margin could increase to $49,9 \%$ if producers adopt the maximum utility plan and up to $62,6 \%$ if they adopt the maximum return plan, given the constraints in factors of production, the technical and economic standards of production alternatives and the targeted level of risk.

AEA Code: 716

Key Words: economic efficiency frontier, risk, linear programming

M.Sc. em Economia Aplicada pela ESALQ/USP e Engenheiro Agrônomo da Empresa de Pesquisa e Extensão Rural de Santa Catarina. Artigo elaborado a partir de sua dissertação de Mestrado.

"'Professor Associado do Departamento de Economia e Sociologia Rural da ESALQ/USP.

\begin{tabular}{|l|l|l|l|l|}
\hline ANÁLISE ECONÔMICA & ANO 16 & N. 29 & SETEMBRO/98 & p. 91-110 \\
\hline
\end{tabular}




\section{1 - INTRODUÇÃO}

O objetivo principal deste artigo é a análise da eficiência econômica de empresas agrícolas sob condições de risco, através de um modelo de fronteira. Desvios da empresa em relação à fronteira têm uma interpretação natural como medida de eficiência, sendo as informações sobre a estrutura da fronteira e sobre a eficiência relativa das unidades econômicas de grande interesse prático.

A Empresa de Pesquisa e Extensão Rural do Estado de Santa Catarina (EPAGRI S.A), que conta atualmente com uma rede contábil de aproximadamente 500 empresas agrícolas monitoradas, objetiva obter os padrões técnicos e econômicos das atividades agrícolas e dos principais sistemas de produção. Torna-se assim importante simular cenários para averiguar os resultados dessas empresas, caso fossem adotados os padrões mais adequados para sua disponibilidade de recursos. $O$ uso de referências e padrões é corrente em países de agricultura avançada, sendo que essa prática pode ser incorporada à agricultura catarinense baseandose, por exemplo, em resultados gerados por modelos de programação linear.

Os resultados deste estudo poderão ser bastante úteis para o tomador de decisão, para os setores que interagem direta ou indiretamente com a empresa, para os políticos e instituições regulamentadoras e para outros agentes interessados no assunto. Eficiência é um conceito importante para a determinação da viabilidade competitiva das empresas, devendo os tomadores de decisão estarem informados sobre o seu nível de ineficiência em relação aos seus competidores no mercado e de sua posição em relação à fronteira de possibilidades técnico-econômicas. Além disso, a metodologia proposta neste estudo pode facilitar a análise de eficiência das empresas agrícolas ao introduzir o elemento risco no modelo.

Objetiva-se estimar a fronteira de eficiência econômica utilizando-se da programação linear, incorporando o risco no modelo, e dispondo-se das evidências empíricas a partir do tratamento de dados cross-section do Sul do Estado de Santa Catarina. Consideram-se, na construção do modelo, as principais alternativas de mercado e de padrões de desempenho técnico e econômico da região, assim como a disponibilidade individual de recursos das empresas.

Os objetivos específicos deste estudo incluem a identificação, ao longo da fronteira de eficiência econômica, do ponto de maximização da utilidade e da margem bruta. Para a maximização da utilidade, assume-se o ponto na fronteira no qual a distância é mínima em relação à situação atual da empresa. 


\section{2 - O MODELO TEÓRICO ADOTADO: MOTAD}

Hazel (1971), introduz risco no modelo determinista convencional de programação linear, através do uso de uma aproximação linear (MOTAD minimização do desvio absoluto total), ao chamado enfoque da médiavariância de Markovitz (1952). Apresenta o modelo MOTAD como um substituto para a programação quadrática, que teria como propósito a determinação do grupo de planos possiveis que estabelecessem a relação entre a variância $V$ e um nivel de renda esperada $E$. Tais planos englobam os chamados pares de eficiência E-V e definem uma fronteira eficiente sobre todos os grupos de planos possíveis, conforme ilustra a Figura 1.

A fronteira eficiente, gerada pelo MOTAD, pode ser definida como o lugar geométrico dos pontos correspondentes ao mínimo risco necessário para se atingir determinado valor da função lucro, dadas as limitações de recursos das empresas. O produtor pode, de acordo com a teoria microeconômica da utilidade, selecionar o plano mais eficiente de riscorenda para a empresa entre todos os possíveis planos.

Na Figura 1, a inclinação da curva de indiferença no ponto $P$, onde $o$ plano é ótimo, ilustra o conceito do coeficiente de aversão ao risco $\phi$. Para o produtor neutro em relação ao risco, seu mapa das curvas de indiferença será formado por linhas horizontais. Neste caso, a curva de indiferença que corresponde à máxima utilidade passa pelo ponto $Q$ que representa a margem bruta máxima. Portanto a abordagem tradicional de máximo lucro é justamente um caso particular da fronteira $E-V$, onde assume-se a neutralidade ao risco. 
Curvas de Iso-utilidade

E

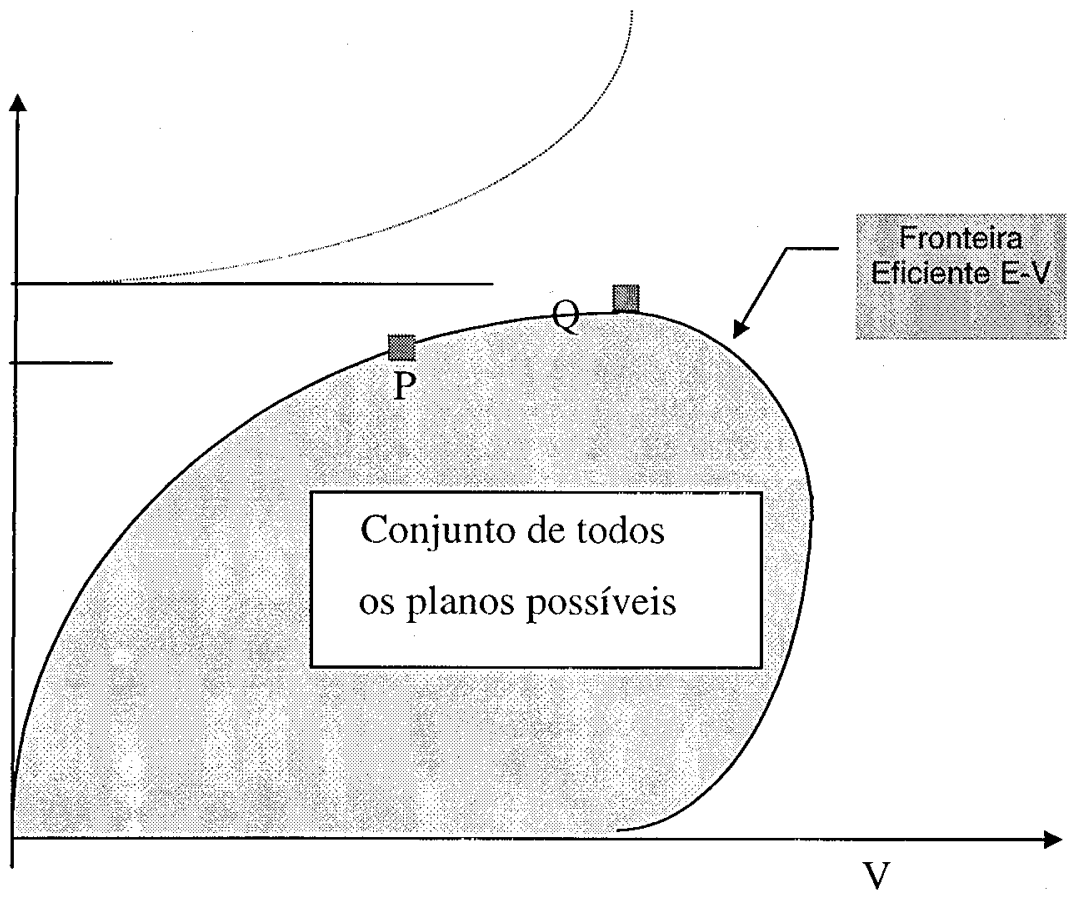

Figura 1 - A fronteira E-V como plano ótimo da empresa (Hazel,1971).

O MOTAD tem sido um método comumente utilizado para avaliação do risco. Vários trabalhos têm sido publicados no exterior e no Brasil utilizandose deste método, tais como os de Bouzit et al. (1994), Oglethorpe (1993), Garcia (1989), Azevedo Filho et al. (1984), Peres (1984), Cruz (1984) e Peres (1981).

Pretende-se assim maximizar uma função objetivo linear, sujeita a um conjunto de restrições também lineares, apresentadas nas expressões (2.1), (2.2), (2.3), (2.4) e (2.5).

$$
\operatorname{Maximizar} \pi=\sum_{j=1}^{n} f_{j} x_{j}
$$

sujeito a: 


$$
\begin{array}{lc}
\sum_{j=1}^{n} a_{i j} x_{j} \leq b_{i} & \text { (para todo } i=1, \ldots, m) \\
\sum_{j=1}^{n} d_{i j} x_{j}+y_{h}^{-} \geq 0 & \text { (para todo } h=1, \ldots, s) \\
x_{j} \geq 0 & \text { (para todo } j=1, \ldots, n \text { ) } \\
\sum_{j=1}^{n} y_{h}^{-} \leq \alpha & (\alpha=0, \ldots \infty)
\end{array}
$$

onde: $x_{j}$ é o nível da j-ésima atividade; $f_{j}$ é a margem bruta esperada da j-ésima atividade; $a_{i j}$ é o coeficiente técnico da j-ésima atividade para o i-ésimo recurso (ou restrição); $b_{i}$ são os níveis de fatores limitantes ou da i-ésima restrição; $n$ número de atividades; $m$ número de restrições; $d_{i j}$ é o desvio da média da margem bruta no i-ésimo ano para a j-ésima atividade; $\mathrm{y}_{\mathrm{h}}$ é uma variável auxiliar que mede a soma dos desvios negativos, podendo assumir os seguintes valores:

$$
\left\{\begin{array}{l}
-1\left(\sum_{\mathrm{i}=1}^{\mathrm{n}} \mathrm{d}_{\mathrm{ij}} \mathrm{x}_{\mathrm{i}}\right), \text { se } \sum_{\mathrm{i}=1}^{\mathrm{n}} \mathrm{d}_{\mathrm{ij}} \mathrm{x}_{\mathrm{i}} \leq 0 \quad \alpha \text { é um escalar. } \\
0, \text { caso contrário; }
\end{array}\right.
$$

Pode-se, facilmente, eliminar o risco do modelo MOTAD, desconsiderando-se as expressões (2.2.3) e (2.2.5). Os dois cenários alternativos, a serem definidos posteriormente, foram formalizados levando em conta este procedimento.

\section{1 - Especificação de dados e procedimentos}

Os dados a serem utilizados, do tipo cross-section, foram obtidos a partir de uma amostra aleatória de 33 empresas agrícolas situadas na região de Tubarão - Sul de Santa Catarina - acompanhadas pela EPAGRI S.A. (Empresa de Pesquisa Agropecuária e Extensão Rural de Santa Catarina), no ano agrícola 94/95. Através do software CONTAGRI, obtém-se em torno de 250 índices técnicos e econômicos, por empresa, para fins de diagnóstico e planejamento.

Tendo-se definido a utilização do modelo de programação matemática MOTAD, conforme especificado nas equações (2.1) a (2.5), serão concebidos dois cenários a serem tomados como referenciais para análise, descritos a seguir: 
- Cenário A: $\phi=0$, onde $\phi=\mathrm{TMS}_{x y}=\frac{d\left(y_{t}\right)}{d\left(x_{t}\right)}$ corresponde ao coeficiente de aversão ao risco, implicando que o segundo momento da distribuição da margem bruta não afeta a decisão, ou seja, ignora-se o comportamento risco-averso do produtor no modelo de planejamento da empresa. A solução ótima é determinada maximizando-se a margem bruta esperada;

- Cenário B: $\phi$ é positivo, incorporando-se assim o comportamento risco-averso do produtor no modelo de planejamento da empresa. Este cenário é o caso padrão de análise E-A. A solução de equilíbrio é obtida maximizando-se a utilidade esperada do produtor.

Para identificar o plano ótimo, objetivando-se o máximo retorno ou utilidade ao longo da fronteira, assumiu-se as seguintes pressuposições: a) a margem bruta (retorno) é função monotonamente decrescente do desvio absoluto (risco); b) a função utilidade do produtor tem somente dois argumentos: a média e o desvio absoluto da margem bruta; c) os produtores buscam operar sobre a fronteira de eficiência no ponto de menor distância em relação à situação de retorno e risco atual, desde que conheçam a função de densidade de probabilidade, com média e variância finita, dos valores futuros das margens brutas de cada atividade com potencial para entrar no plano.

Para a caracterizeção da função objetivo e das restrições do desvio em relação à média das margens brutas, de terra, de trabalho e de capital, visando à definição da situação atual e de pontos ao longo da fronteira de eficiência econômica, sugere-se ao leitor remeter-se à dissertação original do primeiro autor.

Considerando o período de 1988-89 a 1994-95, foram obtidas médias das margens brutas para cada atividade, conforme apresentado na Tabela 1. Com base nestes valores foram obtidos os desvios em relação à média para cada atividade, em cada ano. 
Tabela 1 - Padrões de margem bruta das atividades para 1989 a 1995.

\begin{tabular}{|c|c|c|c|c|c|c|c|}
\hline Atividade & 1989 & 1990 & 1991 & 1992 & 1993 & 1994 & 1995 \\
\hline FumoA & 2910.67 & 2057.98 & 3327.03 & 3237.15 & 3304.87 & 2931.33 & 3313.43 \\
\hline FumoB & 2259.72 & 2057.98 & 2506.12 & 2211.54 & 2604.15 & 2231.76 & 2180.19 \\
\hline FumoC & 1767.52 & 2057.98 & 1316.03 & 1020.59 & 1770.58 & 1401.21 & 1190.57 \\
\hline BoviAll & 438.61 & 464.42 & 246.86 & 187.86 & 309.96 & 296.1 & 443.38 \\
\hline BoviBII & 145.74 & 146.18 & 109.99 & 86.07 & 166.98 & 132.24 & 186.10 \\
\hline BoviCil & -59.41 & 35.29 & 24.62 & 17.13 & 58.26 & 16.27 & 68.61 \\
\hline BoviAlll & 438.61 & 464.42 & 246.86 & 187.86 & 309.96 & 296.1 & 443.38 \\
\hline BoviBll! & 145.74 & 146.18 & 109.99 & 86.07 & 166.98 & 132.24 & 186.10 \\
\hline BoviClil & -59.41 & 35.29 & 24.62 & 17.13 & 58.26 & 16.27 & 68.61 \\
\hline MilhoA & 915.84 & 496.63 & 426.14 & 401.52 & 508.53 & 545.74 & 483.98 \\
\hline MilhoB & 504.91 & 154.12 & 286.36 & 293.32 & 370.59 & 332.90 & 293.60 \\
\hline MilhoC & 283.41 & 13.99 & 172.30 & 208.62 & 236.53 & 155.79 & 144.76 \\
\hline SuinoA & 461.41 & 839.09 & 273.51 & 273.27 & 867.94 & 614.41 & 702.65 \\
\hline SuinoB & 311.12 & 610.47 & 181.46 & 142.28 & 638.20 & 407.84 & 443.06 \\
\hline SuinoC & -15.11 & -21.68 & -21.33 & 12.64 & 191.89 & 94.57 & 79.19 \\
\hline FeijSA & 406.47 & 810.70 & 250.02 & 158.71 & 347.35 & 588.15 & 698.00 \\
\hline FeijSB & 318.49 & 546.76 & 250.02 & 158.71 & 347.35 & 399.52 & 570.00 \\
\hline FeijSC & 247.33 & 334.72 & 250.02 & 158.71 & 347.35 & 240.99 & 385.00 \\
\hline PeixeA & 961.14 & 1249.05 & 558.44 & 1076.50 & 2595.38 & 1720.00 & 2006 \\
\hline PeixeB & 627.37 & 712.58 & 558.44 & 611.71 & 1590.55 & 825.00 & 1013 \\
\hline PeixeC & 467.66 & 712.58 & 558.44 & 132.62 & 544.44 & 229.00 & 317 \\
\hline FeijAA & 376 & 687.39 & 300.38 & 375.84 & 406.39 & 595.03 & 522.58 \\
\hline FeijAB & 280 & 492.41 & 300.38 & 249.66 & 230.10 & 327.59 & 371.11 \\
\hline FeijAC & 184 & 226.87 & 300.38 & 122.95 & 136.26 & 85.67 & 175.05 \\
\hline Horta & 511 & 511 & 511 & 511 & 511 & 511 & 511 \\
\hline CCG & -0.18 & -0.18 & -0.18 & -0.18 & -0.18 & -0.18 & -0.18 \\
\hline
\end{tabular}

Obs.: As variáveis $x_{j}$ (vide expressão 2.1), que correspondem às atividades, estão em US $\$$ e foram codificadas da seguinte forma: FumoA = atividade Fumo-estufa com padrão técnico-econômico $A$ (o padrão é identificado pela última letra maiúscula); BoviB = atividade Bovinocultura com padrão técnico-econômico B; MilhoC = atividade Milho Safra com padrão técnico-econômico $\mathrm{C}$; SuinoA = atividade Suinocultura com padrão técnico-econômico $A$; FeijSC = atividade Feijão Safra com padrão técnico-econômico $C$; PeixeA = atividade Piscicultura com padrão técnico-econômico $A$; FeijAB = atividade Feijāo Adicional com padrão técnico-econômico B; Horta = atividade Quintal Doméstico; CCG = atividade de compra de Capital de Giro; FF = fator que restringe a área plantada de Fejjão Adicional até no máximo à área plantada de Fumo-estufa.

Fonte: EPAGRI S.A.

A Tabela 2 mostra os coeficientes técnicos e restrições de terra, trabalho e capital impostos às atividades. 
Tabela 2 - Matriz de coeficientes

técnicos e restrições impostas às atividades.

\begin{tabular}{|c|c|c|c|c|c|c|c|c|c|c|c|c|c|c|}
\hline & $T_{1}$ & $T_{2}$ & $T_{3}$ & $\mathrm{QL}$ & $P$ & $M_{1}$ & $M_{2}$ & $M_{3}$ & $M_{4}$ & $\mathrm{M}_{5}$ & $M_{6}$ & $\overline{C G}$ & $\mathrm{CC}$ & $\mathrm{FF}$ \\
\hline FumoA & 1 & 0 & 0 & 0 & 0 & 7 & 26 & 40 & 40 & 16 & 3 & 877.75 & $\overline{0}$ & -1 \\
\hline FumoB & 1 & 0 & 0 & 0 & 0 & 7 & 26 & 40 & 40 & 16 & 3 & 870.11 & 0 & -1 \\
\hline FumoC & 1 & 0 & 0 & 0 & 0 & 7 & 26 & 40 & 40 & 16 & 3 & 998.54 & 0 & -1 \\
\hline BoviAll & 1 & 0 & 1 & 0 & 0 & 3.3 & 3.3 & 3.3 & 3.3 & 4 & 5.8 & 77.79 & 0 & 0 \\
\hline BoviBII & 1 & 0 & 1 & 0 & 0 & 3.3 & 3.3 & 3.3 & 3.3 & 4 & 5.8 & 84.20 & 0 & 0 \\
\hline BoviCII & 1 & 0 & 1 & 0 & 0 & 3.3 & 3.3 & 3.3 & 3.3 & 4 & 5.8 & 83.18 & 0 & 0 \\
\hline BoviAlli & 0 & 1 & 0 & 0 & 0 & 3.3 & 3.3 & 3.3 & 3.3 & 4 & 5.8 & 77.79 & 0 & 0 \\
\hline BoviBIII & 0 & 1 & 0 & 0 & 0 & 3.3 & 3.3 & 3.3 & 3.3 & 4 & 5.8 & 84.20 & 0 & 0 \\
\hline Boviclll & 0 & 1 & 0 & 0 & 0 & 3.3 & 3.3 & 3.3 & 3.3 & 4 & 5.8 & 83.18 & 0 & 0 \\
\hline MilhoA & 1 & 0 & 0 & 0 & 0 & 7 & 7 & 5 & 6 & 8 & 0 & 125.89 & 0 & 0 \\
\hline MilhoB & 1 & 0 & 0 & 0 & 0 & 6 & 6 & 5 & 5 & 7 & 0 & 133.61 & 0 & 0 \\
\hline MilhoC & 1 & 0 & 0 & 0 & 0 & 6 & 6 & 5 & 4 & 6 & 0 & 145.30 & 0 & 0 \\
\hline SuinoA & 0 & 0 & 0 & 0 & 0 & 3 & 3 & 3 & 3 & 3 & 3 & 1020.8 & 0 & 0 \\
\hline SuinoB & 0 & 0 & 0 & 0 & 0 & 3 & 3 & 3 & 3 & 3 & 3 & 1033.3 & 0 & 0 \\
\hline SuinoC & 0 & 0 & 0 & 0 & 0 & 3 & 3 & 3 & 3 & 3 & 3 & 1510.0 & 0 & 0 \\
\hline FeijSA & 1 & 0 & 0 & 0 & 0 & 6 & 7 & 12 & 6 & 0 & 0 & 62.19 & 0 & 0 \\
\hline FeijSB & 1 & 0 & 0 & 0 & 0 & 6 & 7 & 12 & 6 & 0 & 0 & 56.14 & 0 & 0 \\
\hline FeijSC & 1 & 0 & 0 & 0 & 0 & 6 & 7 & 12 & 6 & 0 & 0 & 51.59 & 0 & 0 \\
\hline PeixeA & 0 & 1 & 0 & 0 & 1 & 1 & 2 & 2 & 15 & 2 & 0 & 56.00 & 0 & 0 \\
\hline PeixeB & 0 & 1 & 0 & 0 & 1 & 0 & 0 & 1 & 10 & 1 & 0 & 56.00 & 0 & 0 \\
\hline PeixeC & 0 & 1 & 0 & 0 & 1 & 0 & 0 & 0 & 5 & 0 & 0 & 42.00 & 0 & 0 \\
\hline FeijAA & 0 & 0 & 1 & 0 & 0 & 0 & 0 & 0 & 4 & 4 & 10 & 77.72 & 0 & 1 \\
\hline Feij $A B$ & 0 & 0 & 1 & 0 & 0 & 0 & 0 & 0 & 4 & 4 & 10 & 8.48 & 0 & 1 \\
\hline FeijAC & 0 & 0 & 1 & 0 & 0 & 0 & 0 & 0 & 4 & 4 & 10 & 87.29 & 0 & 1 \\
\hline Horta & 1 & 0 & 1 & 1 & 0 & 19 & 24 & 22 & 22 & 18 & 14 & 0 & 0 & 0 \\
\hline CCG & 0 & 0 & 0 & 0 & 0 & 0 & 0 & 0 & 0 & 0 & 0 & -1 & 1 & 0 \\
\hline
\end{tabular}

Obs.: Os fatores representados no modelo, a partir dos quais o vetor $b$ (vide expressão 2.2) foi composto, foram os seguintes: $T_{1}=$ disponibilidade do tipo de solo Classe II (aptidão para cultivo de todas as culturas) de cada empresa para uso com cultura principal; $T_{2}=$ disponibilidade do tipo de solo Classe III (aptidão para cultivos perenes, tais como, pastagem e Piscicultura) de cada empresa para uso com cultura principal; $\mathrm{T}_{3}=$ disponibilidade do tipo de solo Classe IIA de cada empresa para uso com cultura adicional; $\mathrm{QL}=$ fator que permite impor certa quantidade da superfície agrícola util para uso de horta e pomar doméstico; $P$ = fator que impõe limite à produção de peixes; $M_{1}$ a $M_{6}=$ disponibilidade em cada bimestre de mão-de-obra familiar; $C G=$ disponibilidade de capital de giro próprio; $\mathrm{CC}=$ possibilidade de compra de capital de giro; e FF = área máxima de Feijão adicional em função da área plantada de Fumo estufa.

\section{2 - Definição da situação atual das empresas e a geração da fronteira de eficiência}

Para proceder a análise da situação atual, optou-se em tipificar o agregado de empresas em função da origem da renda bruta total (RBT). Os critérios para executar a classificação das empresas foram os mesmos adotados pelo software CONTAGRI, que são os seguintes: a) Grupos com uma atividade predominante - quando mais de $60 \%$ da renda bruta total provêm de apenas uma atividade; b) Grupos com duas atividades predominantes - quando duas atividades combinadas correspondem a $75 \%$ 
ou mais da Renda bruta total e nenhuma delas representar, isoladamente, menos de $30 \%$; c) Grupos com três atividades predominantes - quando três atividades combinadas totalizarem mais de $80 \%$ da renda bruta total e nenhuma delas representar, isoladamente, menos de $20 \%$; d) Grupos sem orientação técnico-econômica definida - quando o grupo não se enquadrar em nenhum dos três tipos anteriores.

A margem bruta total foi obtida multiplicando-se o dimensionamento de cada atividade pelo padrão $A, B$ ou $C$ de margem bruta esperada que melhor se ajustasse à situação da empresa no ano agrícola de 1994-95. Naturalmente, o enquadramento da atividade em determinado padrão tem influências na magnitude encontrada da margem bruta e de seu desvio absoluto.

O modelo de programação matemática utilizado para gerar a fronteira eficiente foi apresentado nas equações (2.1) a (2.5). Note-se que a equação (2.5) necessita somente ser parametrizada para valores de $\alpha \leq \alpha^{*}$, sendo que $\alpha^{\star}$ é a solução do modelo de programação linear determinístico. Para valores de $\alpha$ maiores do que $\alpha^{*}$ não existe solução possivel.

Para gerar a fronteira de eficiência, primeiramente, informa-se a disponibilidade de fatores (RHS) e, a seguir, encontra-se a solução do modelo de programação linear simples através das equações (2.1), (2.2) e (2.4). O valor encontrado serviu de referência à parametrização feita pelo modelo completo MOTAD que foi programado através da linguagem de otimização GAMS (General Algebraic Modelling System).

\section{3 - Estimativa do "ponto ótimo" e do grau de aversão ao risco}

Como visto anteriormente, para o atual mix de atividades de cada empresa correspondem um nível de desvio absoluto $(x)$ e um nível de margem bruta esperada (y), conforme mostra a Figura 2.

Com o propósito de estimar o ponto de equilíbrio $z\left(x_{1}, y_{1}\right)$ assumiu-se que este ponto fosse aquele mais próximo do ponto $s\left(x_{2}, y_{2}\right)$ em relação à fronteira de eficiência. O plano ótimo, ou de equilíbrio, $z\left(x_{1}, y_{1}\right)$, é, portanto, o ponto na fronteira no qual a distância (d) para s $\left(\mathrm{x}_{2}, \mathrm{y}_{2}\right)$ é mínima.

Tais pressupostos estão de acordo com as hipóteses assumidas por Peres (1976), a menos da consideração de que os pares de valores $\left(x_{2}, y_{2}\right)$ estivessem sobre a fronteira de eficiência (vide Figura 2). Assumiu-se ainda, que a curva de indiferença era uma linha reta que passava através do ponto $\left(\mathrm{x}_{1}, \mathrm{y}_{1}\right)$ e tangente à fronteira; e que o trade-off entre risco e retorno era constante em todo espaço bi-dimensional retorno-risco. 


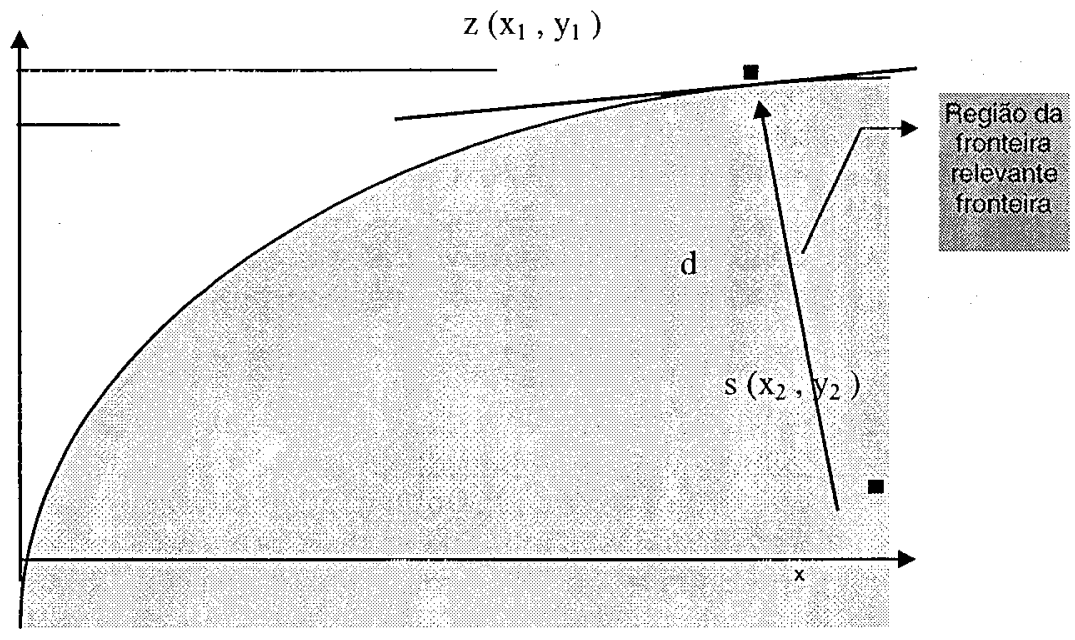

Figura 2 - Determinação do ponto de equilíbrio através da distância euclidiana do ponto correspondente à situação atual até a fronteira de eficiência relevante.

O primeiro procedimento para obtenção do ponto de equilibrio foi o de expressar algebricamente os pontos obtidos na fronteira de eficiência através da parametrização.

O modelo estatístico mostrado na equação (2.6) é uma forma funcional Cobb-Douglas que relaciona retorno $(y)$ e risco $(x)$ possivel, sendo este modelo não-linear nos parâmetros $\alpha$ e $\beta$.

$$
\mathrm{y}=\alpha \mathrm{x}^{\beta} e
$$

onde: y é a margem bruta esperada ao longo da fronteira de eficiência; $x$ o nível de desvio absoluto; $\alpha$ um parâmetro; $\beta$ um parâmetro que corresponde à elasticidade de substituição entre retorno e risco; e e o resíduo do modelo.

O comportamento desta função foi mostrado na Figura 2 , assumindo-se que $\beta<1$. Um aspecto positivo deste modelo é que pode ser reescrito na forma linear. A relação não-linear entre os parâmetros $\alpha$ e $\beta$ é, portanto, transformada para uma relação linear nos parâmetros. Assim sendo, o estimador dos mínimos quadrados ordinários pode ser utilizado. 
O cálculo do ponto na fronteira para o qual a distância (d) é mínima foi obtido por meio da função euclidiana. Segundo Chiang (1982), a distância euclidiana (d) é definida como:

$$
d=\sqrt{\left(x_{2}-x_{1}\right)^{2}+\left(y_{2}-f\left(x_{1}\right)\right)^{2}}
$$

onde o ponto $z$, formado pelo conjunto ordenado $\left(x_{1}, f\left(x_{1}\right)\right)$, e o ponto $s$, pelo conjunto ordenado $\left(x_{2}, y_{2}\right)$, os quais foram mostrados anteriormente na Figura 2.

Assim sendo, pode-se encontrar os valores ao longo da fronteira $\left(x_{1}\right.$, $f\left(x_{1}\right)$ ) para os quais a distância é mínima e que correspondem ao ponto ótimo para o cenário $B$.

A Taxa Marginal de Substituição entre a margem bruta e o desvio absoluto é dada por $\phi=\operatorname{TMS}_{\mathrm{xy}}=\frac{d\left(y_{t}\right)}{d\left(x_{t}\right)}$, ou seja, $\phi$ é o coeficiente de aversão ao risco. Se o produtor é avesso ao risco, então, $\phi>0$ e a curva de iso-utilidade linear possui inclinação positiva no espaço y, $x$. Segundo Hazel e Norton (1986), $\phi$ é um número com interpretação intuitiva, sendo que o valor de $\phi=1,65$, por exemplo, corresponde ao nivel de aversão ao risco tolerado pelos estatísticos para o teste de confiança de $5 \%$ sobre a hipótese selecionada, caso a margem bruta tenha distribuição normal.

\section{3 - RESULTADOS EMPÍRICOS E DISCUSSÃO}

Os resultados obtidos da aplicação empírica do modelo proposto são apresentados e analisados a seguir. Inicialmente, caracteriza-se a situação atual das empresas estudadas em termos de retorno e de risco e, para depois, mostrar-se a estimativa da eficiência econômica, comparando-se os resultados obtidos com os de outros estudos sobre o assunto.

\section{1 - Caracterização da situação atual}

As 33 empresas analisadas possuem sistemas de produção diferenciados, apesar de se localizarem na mesma região. Seguindo os critérios de classificação de empresas definidos seção 2.2, obteve-se a agregação de oito tipos de empresas, em função da origem da renda bruta total, que está apresentada na Tabela 3.

A Tabela 3 mostra que o tipo de empresa Bovino (a) possui, comparativamente aos demais, um nível de margem bruta menor (US\$ $7.129,21$ ) e um nível de desvio absoluto maior (US\$ 5.818,78). No que diz respeito a este último valor, foi um resultado de certa forma surpreendente, visto que a atividade Bovinocultura é considerada tradicional na região. A 
explicação para a magnitude do desvio absoluto deve estar relacionada ao tamanho da superfície forrageira principal (SFP) e ao padrão tecnológico das atividades que fazem parte deste grupo de empresas.

Tabela 3 - Sistemas de produção para o agregado das 33 empresas e seus respectivos níveis de margem bruta e desvio absoluto (US $\$$ ).

\begin{tabular}{l|r|r|r}
\hline Tipo de Empresa & \% do total & Desvio absoluto & margem bruta \\
\hline Em função da Renda bruta total: & & & \\
Bovino (a1) & 33 & 5170,82 & 6321,91 \\
Bovino e Suinos ciclo completo (a2) & 9 & 8194,67 & 10089,33 \\
Fumo estufa (b1) & 9 & 2776,33 & 8948,00 \\
Fumo estufa e Suinos (b2) & 6 & 3719,50 & 10006,50 \\
Fumo estufa e Bovino (b3) & 12 & 2294,75 & 6377,25 \\
Suínos ciclo completo e Bovino (c1) & 9 & 5228,67 & 6316,33 \\
Suínos, Fumo e Bovino (c2) & 6 & 5825,00 & 10551,50 \\
Sem orientação econômica (d) & 15 & 4076,40 & 8702,80 \\
Em função da atividade principal: & 42 & & \\
Bovino (a) & 27 & 5818,79 & 7129,21 \\
Fumo estufa (b) & 15 & 2771,89 & 8040,67 \\
Suínos (c) & 15 & 5467,20 & 8010,40 \\
Sem orientação econômica (d) & 100 & 4076,40 & 8702,80 \\
Total & & 4670,55 & 7749,73 \\
\hline
\end{tabular}

O tipo de empresa Fumo-estufa apresentou, em média, para cada US\$ 1,00 de retorno um nível de risco de US $\$ 0,34$, sendo que dentre os tipos de empresas analisadas foi aquela que obteve menor nível de desvio absoluto (US\$ 2.771,88).

A dispersão dos níveis de risco e de retorno tomados pelas 33 empresas analisadas é mostrado na Figura 3. Observaram-se valores médios do desvio absoluto de US\$4.670,55 por empresa (máximo de US\$ $13.167,00$ e mínimo de US $\$ 1.493,00$ ), com o desvio padrão de US\$ 3.334,50. Para o retorno, observaram-se valores médios da margem bruta de US $\$ 7.613,00$ (máximo de US $\$ 14.620,00$ e mínimo de US\$1.769,00), com desvio padrão de US\$3.284,00. 
20000

15000

10000

5000

0

$\begin{array}{llllllll}0 . & 2000 & 4000 & 6000 & 8000 & 10000 & 12000 & 14000\end{array}$

Desvio Absoluto (US\$)/Empresa

Figura 3 -. Caracterização da situação atual em

termos de margem bruta e desvio absoluto para as empresas.

Analisando-se a Figura 3 , não se pode afirmar, por exemplo, que a empresa 54 seja três vezes mais eficiente que a empresa 28 , dado que seus níveis de risco são aproximadamente iguais. Seria mais interessante que a comparação fosse feita em relação à fronteira de possibilidade individual da empresa para se definir a eficiência relativa ao seu nível de risco. Portanto é perfeitamente possivel que as empresas 54 e 28 tenham a mesma eficiência econômica relativa, embora, em termos absolutos, a margem bruta destas empresas tenham magnitudes completamente diferentes.

\section{2 - Eficiência econômica para maximização da utilidade e do retorno}

O plano ótimo foi definido considerando-se o objetivo comportamental da unidade produtiva, ou seja, apresentando neutralidade ou aversão ao risco. A eficiência econômica foi medida comparando-se a margem bruta observada pela empresa com o valor da margem bruta ótima.

A Figura 4 mostra um potencial de crescimento econômico diferente para as empresas estudadas, mesmo assumindo-se conhecimento perfeito das alternativas e dos padrões de margem bruta esperados e de sua variabilidade. Os resultados sugerem diferenças em termos de disponibilidade de fatores por parte destas empresas. 


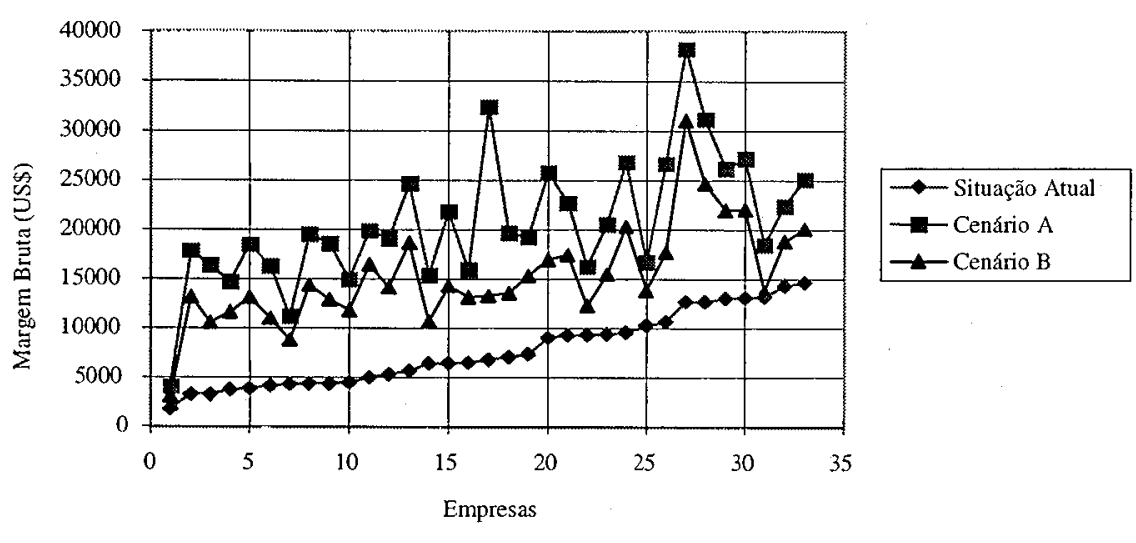

Figura 4 - Potencial de crescimento econômico para o comportamento maximizador da margem bruta (cenário A) e da utilidade (cenário B).

Em média, as empresas analisadas apresentaram uma eficiência econômica de $50,1 \%$ e de $37,4 \%$, considerando-se o objetivo de maximizar a utilidade e maximizar a margem bruta, respectivamente. Assim sendo, os resultados indicam um potencial de aumento do retorno das empresas médio de $49,9 \%$ e $62,6 \%$ quando o produtor adotar, respectivamente, o plano de máxima utilidade e de máximo retorno dada a disponibilidade de recursos, os padrões técnicos e econômicos e as alternativas de produção.

A representação do ponto da situação atual, da distância euclidiana e da fronteira de eficiência relevante, conforme já mostrados na Figura 2, tem suas estimativas apresentadas na Tabela 4 . Os coeficientes de determinação $\left(R^{2}\right)$ das funções Cobb-Douglas estimadas foram acima de 0,95 , o que se constitui num indicativo de muito bom ajuste.

Todas as 33 empresas analisadas localizaram-se abaixo da fronteira de eficiência, como teoricamente era de se esperar, uma vez que o modelo de fronteira utilizado envolve maximização da margem bruta. Entretanto é perfeitamente possivel que algumas empresas estivessem sobre ou acima da fronteira, uma vez que estão sendo utilizados padrões econômicos e coeficientes técnicos médios para as alternativas potenciais da região. Portanto, considerando-se a natureza do método utilizado e a possibilidade dos coeficientes adotados como padrões não se ajustarem à realidade de uma determinada empresa ou grupo delas, algumas empresas poderiam se localizar acima da fronteira de eficiência. 
Tabela 4 - Estimativa dos parâmetros da fronteira de eficiência na forma Cobb-Douglas, do coeficiente de aversão ao risco e da distância euclidiana para cada uma das empresas.

\begin{tabular}{|c|c|c|c|c|c|c|}
\hline Empresas & $\overline{x_{2}}$ & $y_{2}$ & $\alpha$ & $\beta$ & $\phi$ & $\mathrm{d}$ \\
\hline a1183019 & \begin{tabular}{|c|}
7,7571 \\
\end{tabular} & 8,0907 & 7,9086 & 0,2112 & 1,5953 & 1,4247 \\
\hline a1183024 & 8,0736 & 8,3693 & 8,2602 & 0,1032 & 0,3058 & 0,7202 \\
\hline a1183031 & 7,5601 & 8,0922 & 7,4959 & 0,2436 & 1,7893 & 1,2099 \\
\hline a1183039 & 7,9814 & 8,2276 & 7,4461 & 0,2442 & 1,2304 & 1,1342 \\
\hline a1183060 & 7,6064 & 8,3457 & 7,4316 & 0,2548 & 1,7835 & 0,9923 \\
\hline a1183062 & 7,7824 & 8,2654 & 7,5490 & 0,2584 & 1,9287 & 1,2534 \\
\hline a1186001 & 9,3980 & 9,4796 & 8,7750 & 0,1312 & 0,2561 & 0,5239 \\
\hline a1186002 & 8,7260 & 8,9072 & 8,7183 & 0,1064 & 0,2864 & 0,7353 \\
\hline a1186003 & 9,4855 & 9,5715 & 8,5775 & 0,1338 & 0,1981 & 0,2727 \\
\hline a1186004 & 9,1262 & 9,2360 & 8,2224 & 0,1445 & 0,2270 & 0,3019 \\
\hline a1186005 & 7,3085 & 7,4782 & 6,6291 & 0,1934 & 0,4386 & 0,5540 \\
\hline a2183020 & 8,1741 & 8,4149 & 8,2772 & 0,1666 & 0,5180 & 0,9698 \\
\hline a2183050 & 9,2379 & 9,4505 & 9,2100 & 0,0981 & 0,2503 & 0,6625 \\
\hline a2183063 & 9,2833 & 9,4758 & 9,1666 & 0,0900 & 0,1927 & 0,5241 \\
\hline b1183001 & 8,1403 & 8,7803 & 7,6778 & 0,2267 & 1,0229 & 0,7245 \\
\hline b1183016 & 7,5197 & 8,8677 & 7,5270 & 0,2709 & 2,3786 & 0,6721 \\
\hline b1183054 & 8,0245 & 9,4910 & 8,2243 & 0,1629 & 0,7391 & 0,0399 \\
\hline b2183032 & 8,3925 & 9,1445 & 8,0093 & 0,1685 & 0,4907 & 0,2750 \\
\hline b2183036 & 8,0147 & 9,2733 & 7,6737 & 0,2672 & 1,7857 & 0,5235 \\
\hline b3183009 & 7,4622 & 8,7706 & 7,4220 & 0,2528 & 1,7622 & 0,5214 \\
\hline b3183021 & 7,9603 & 8,6422 & 8,0811 & 0,2280 & 1,9486 & 1,2224 \\
\hline b3183051 & 7,7668 & 8,3834 & 7,6779 & 0,2544 & 2,1053 & 1,2311 \\
\hline b3183053 & 7,7017 & 9,1080 & 6,8503 & 0,3873 & 3,7907 & 0,6762 \\
\hline c1183018 & 8,3680 & 8,5092 & 2,8762 & 0,2059 & 1,0035 & 1,2214 \\
\hline c1183026 & 9,0098 & 9,1690 & 8,0906 & 0,2066 & 0,5995 & 0,7667 \\
\hline c1183028 & 8,0697 & 8,3880 & 7,6298 & 0,2352 & 1,2275 & 1,1095 \\
\hline c2183002 & 7,5924 & 8,7769 & 7,7372 & 0,2474 & 2,1674 & 0,8141 \\
\hline c2183005 & 9,1765 & 9,5901 & 8,7185 & 0,1299 & 0,2804 & 0,3177 \\
\hline d178013 & 7,5999 & 8,8324 & 2,6220 & 0,9891 & 12,6205 & 0,9289 \\
\hline d183004 & 8,0818 & 9,1348 & 8,6736 & 0,1367 & 0,8034 & 0,6376 \\
\hline d183012 & 8,5564 & 9,1523 & 8,8205 & 0,0972 & 0,3036 & 0,4975 \\
\hline d183013 & 7,9617 & 8,5722 & 7,6838 & 0,2188 & 1,3406 & 1,0097 \\
\hline d185001 & 8,8650 & 9,4470 & 8,2411 & 0,2430 & 1,3235 & 0,9214 \\
\hline Média & 8,2353 & 8,8314 & 7,6941 & 0,2215 & 1,4756 & 0,7694 \\
\hline Desvio padrão & 0,6387 & 0,5260 & 1,4120 & 0,1532 & 2,1708 & 0,3441 \\
\hline
\end{tabular}

Obs.: $\mathrm{x}_{2}, \mathrm{y}_{2}$ são os valores em logaritmo das coordenadas da situação atual de cada empresa; $\alpha, \beta$ as estimativas dos parâmetros da fronteira na forma Cobb-Douglas; $\phi$, $d$ refere-se à estimativa do coeficiente de aversão ao risco e da distância euclidiana para cada empresa.

O coeficiente de aversão ao risco $(\phi)$, que corresponde à Taxa Marginal de Substituição entre a margem bruta e o desvio absoluto, teve um valor médio estimado para $\circ$ agregado de empresas de $\phi=1,47$, conforme 
mostra a Tabela 4, o que significa uma curva de iso-utilidade linear com inclinação positiva no espaço $\mathrm{y}, \mathrm{x}$.

Os resultados de eficiência econômica para o comportamento de maximização da margem bruta e da utilidade, considerando-se a classificação dos grupos em função da Renda bruta total, podem ser visualizados na Figura 5.

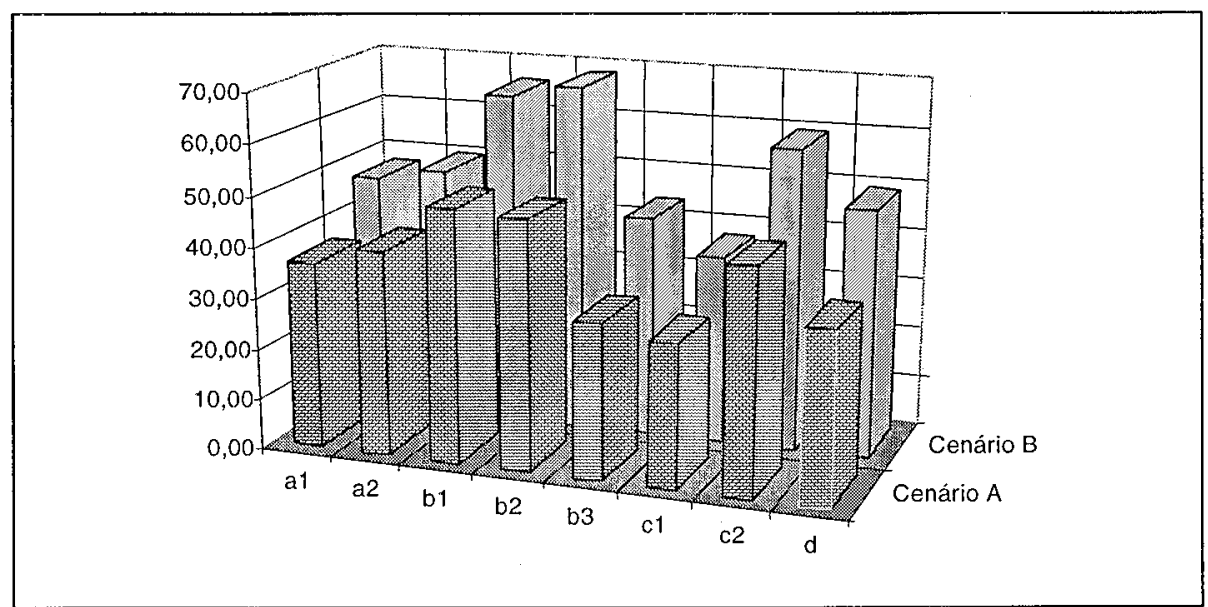

Obs.: a1 = Bovino; a2 = Bovino e Suínos ciclo completo; b1 = Fumo-estufa; b2 = Fumo-estufa e Suínos ciclo completo; $\mathrm{b} 3=$ Fumo-estufa e Bovino; $\mathrm{c} 1$ = Suínos ciclo completo e Bovino; $\mathrm{c} 2$ = Suínos ciclo completo, Fumo e Bovino; $d$ = Sem orientação técnica e econômica definida).

Figura 5 - Eficiência Econômica Relativa estimadas para o comportamento maximizador da margem bruta e da utilidade para os diferentes tipos de empresas analisadas

Os tipos de empresas predominantemente Fumo-estufa e predominantemente Fumo-estufa e Suínos ciclo completo foram os que apresentaram maior eficiência, 65,91\% e 68,27\%, respectivamente, para o comportamento de maximização da utilidade e, 49,55\% e 48,76\%, respectivamente, para o comportamento de maximização da margem bruta. Ao contrário, os tipos de empresas predominantemente Suínos ciclo completo e predominantemente Fumo-estufa e Bovino mostraram-se, respectivamente, menos eficientes com $37,13 \%$ e $43,56 \%$ para maximização da utilidade e, $28,11 \%$ e $30,55 \%$, para o comportamento maximizador da margem bruta.

Os sistemas de produção com atividade principal Fumo-estufa (b), conforme mostra a Tabela 5, foram os que apresentaram maior eficiência para os dois cenários, enquanto que os menos eficientes foram os sistemas 
de produção com atividade principal Suínos ciclo completo e sem orientação técnica econômica definida. Assim sendo, apesar dos sistemas de produção com atividade principal Bovinos terem, em média, uma margem bruta menor (US\$ 7.129,21) na situação atual, esses tipos de empresas apresentaram um nível relativo de eficiência intermediário quando comparados aos demais sistemas de produção.

Tabela 5 - Eficiência econômica relativa para os diferentes tipos de empresas analisadas considerando-se os cenários A e B.

\begin{tabular}{l|c|c}
\hline & Cenário A & Cenário B \\
\hline Em função da Renda bruta total: & 0,37 & 0,47 \\
Bovino (a1) & 0,40 & 0,50 \\
Bovino e Suínos ciclo completo (a2) & 0,50 & 0,66 \\
Fumo estufa (b1) & 0,49 & 0,68 \\
Fumo estufa e Suínos ciclo completo (b2) & 0,31 & 0,44 \\
Fumo estufa e Bovino (b3) & 0,28 & 0,37 \\
Suínos ciclo completo e Bovino (c1) & 0,44 & 0,59 \\
Suínos ciclo completo, Fumo e Bovino (c2) & 0,34 & 0,49 \\
Sem orientação técnica e econômica (d) & & \\
Em função da atividade principal: & 0,38 & 0,48 \\
Bovino (a) & 0,41 & 0,57 \\
Furno estufa (b) & 0,34 & 0,46 \\
Suínos (c) & 0,34 & 0,49 \\
Sem orientação técnica e econômica (d) & 0,37 & 0,50 \\
Média para total de empresas & & \\
\hline
\end{tabular}

Vários estudos empíricos, tais como Ali e Flinn (1989), Parikh e Shah (1994), Hallam \& Machado (1996) e Wang et al. (1996) suportam a tese da existência de significativas ineficiências econômicas em grupos de empresas agrícolas localizadas em uma mesma região, tal como observado neste trabalho. Com exceção de Parikh e Shah (1994), todas as fronteiras estimadas pelos trabalhos anteriores não incluiam o elemento risco diretamente no modelo.

Alterando-se o objetivo de maximizar o retorno para maximizar a utilidade, obtém-se uma redução média proporcionalmente maior do desvio absoluto em relação à variação obtida com a margem bruta. A diminuição média do retorno esperado $(E)$ foi de $27,58 \%$, enquanto do nível de risco $(A)$ foi de $387,72 \%$. Assim, pode-se ainda afirmar que os produtores comportando-se como maximizadores da margem bruta deverão assumir um risco muito superior ao obtido quando maximizadores da utilidade.

Estes resultados são consistentes com os obtidos por Oglethorpe (1995), que também examinou como a estimativa do plano ótimo da empresa, que tem como objetivo maximizar o lucro, difere daquela descrito pela maximização da utilidade. O estudo utilizou funções de utilidade 
obtidas diretamente de um levantamento de 20 empresas agrícolas do norte da Inglaterra, em conjunto com a fronteira de eficiência geradas pelo MOTAD. Este autor constatou que uma pequena redução da renda esperada gera uma redução proporcionalmente maior da variância da renda, quando o objetivo passa de maximização do lucro para maximização da utilidade. No caso em questão, para uma diminuição média da renda esperada $(E)$ em $5,4 \%$ a diminuição na variância da renda (V) foi de $36,6 \%$.

\section{4 - CONCLUSÕES}

As regiões agrícolas do Estado de Santa Catarina, caracteristicamente, oferecem um razoável mix de alternativas potenciais, competindo por recursos. Dispondo-se de padrões de desempenho técnico-econômico das atividades potenciais da região e de informações da disponibilidade individual de recursos das empresas, o modelo MOTAD parece ser uma abordagem básica e adequada, uma vez que permite que se avalie esses diferentes padrões levando-se em conta considerações do tipo portfólio pertinentes à tomada de decisão. As principais características do MOTAD e que o distingüem, de certa forma, de outros modelos de fronteira, são as seguintes: trata diretamente o problema econômico ao longo da fronteira que é construída no espaço bidimensional retorno-risco; e gera uma fronteira de eficiência econômica individual. Para este modelo, a geração da fronteira de eficiência sob condições de risco pode ser feita através de software diversos, tais como o GAMS, resultando em vantagens operacionais e de tempo.

O principal resultado da análise de eficiência econômica é a existência de um potencial médio de crescimento da margem bruta de $49,9 \%$ e $62,6 \%$, quando o produtor adotar, respectivamente, o plano de máxima utilidade e de máximo retorno, dada a disponibilidade de recursos, os padrões técnicos e econômicos das alternativas de produção e o nível de risco que deseja suportar.

Alterando-se o ponto ótimo de maximização do retorno para o de maximização da utilidade, obteve-se uma redução média proporcionalmente maior do desvio absoluto em relação à variação obtida com a margem bruta. A estimativa média do coeficiente de aversão ao risco foi de $\phi=1,47 \mathrm{e}$, dada a sua distribuição diversa, este não é necessariamente bem representado por um valor médio. Os resultados da análise de eficiência implicam, dados os padrões técnicos e econômicos da região, a promoção de significativa melhora dos resultados de margem bruta obtidos atualmente para os dois cenários definidos.

O grau de eficiência econômica medido pelo MOTAD é muito sensivel à qualidade da informação sobre a disponibilidade de fatores, sobre os 
coeficientes da função objetivo e sobre os coeficientes técnicos das empresas. Além disso, a eficiência econômica de empresas agrícolas não depende somente da disponibilidade de fatores físicos da produção e da variabilidade da margem bruta, tal como modelado. Existe um conjunto de fatores socio-econômicos e culturais que afetam o produtor e a administração destas empresas e, indiretamente, os seus resultados técnicos e econômicos.

As principais limitações deste estudo referem-se também ao baixo número de observações (33 empresas), a utilização de dados cross-section e a pressuposição sobre a forma explícita da função utilidade dos produtores, com somente dois argumentos (média e desvio absoluto), visando-se à determinação do ponto de equilíbrio para os dois cenários definidos anteriormente.

Com relação ao modelo MOTAD, pode-se agregar as seguintes limitações: 1) pressuposição de simetria em relação à probabilidade de ocorrência das margens brutas; 2) escolha arbitrária do desvio absoluto com relação à média como proxy para risco; e 3) analisa o risco somente do lado da margem bruta, desconsiderando-se, por exemplo, as flutuações nos custos dos insumos e na oferta de recursos.

A contribuição deste trabalho justifica-se não somente pelas informações e análises possiveis de serem feitas, mas também, por adaptar e desenvolver um instrumental possível de aplicação para as demais regiões do Estado de Santa Catarina. Especialmente para a região sul catarinense, sugere-se a ampliação da matriz das alternativas de produção que, segundo a EPAGRl (1996), conta com 32 atividades agropecuárias acompanhadas, em função da faixa da área e do número de matrizes, o que representaria uma matriz com 96 padrões técnicos e econômicos. Necessita-se, entretanto, estudar essas alternativas em termos da disponibilidade dos coeficientes técnicos e econômicos ao longo de séries históricas abrangentes.

Finalmente, recomenda-se também que em estudos futuros incorporem-se os investimentos necessários para algumas atividades da região, tais como suinocultura, bovinocultura e fumicultura. É fundamental que essa questão dos investimentos adicionais seja melhor tratada na modelagem, uma vez que eles podem explicar e interferir na definição da fronteira de eficiência e nos resultados decorrentes. 


\section{BIBLIOGRAFIA}

ALI, M; FLINN, J.C. Profit efficiency among basmati rice producers. In Pakistan Punjab. Americam Journal of Agricultural Economics, v. 71, n. 2, p. 303-310, 1989.

ARAUJO, L. A. Fronteira de eficiência econômica sob condições de risco: uma análise da convergência econômica entre empresas agrícolas do Sul de Santa Catarina. 1997, Dissertação (Mestrado), ESALQ-USP, 150p.

AZEVEDO FILHO, A.J.B.V. e PÉRES, F.C. Competitividade da cultura da soja em uma empresa da região de Campinas, SP. In: CONTINI, E.; ARAÚJO, J.D.; OLIVEIRA, A.J. et al. Planejamento da propriedade agricola, modelos de decisão, Brasília, EMBRAPA - DDT, p. 289-300, 1984.

BOUZIT, A.M.; RIEU, T.; RIO,P. Modelling farmers' behavior under risk: a generalized MOTAD application. Les revenus agricoles: efficacife, equite, estabilite. Economie Rurale. 69-73p, 1994.

CHIANG, A. C. Matemática para economistas. São Paulo: McGrawhill/ Ed da USP. 1982.

CRUZ, E. R. Aspectos teóricos sobre incorporação de risco em modelos de decisão. Brasília, EMBRAPA-DEP, 1984.

EMPRESA DE PESQUISA AGROPECUÁRIA E EXTENSÃO RURAL DE SANTA CATARINA S.A. Quadro geral de comparação de grupo da região de Tubarão dos anos agricolas 1988/89 até 1994/95, 1996 (não publicado).

GARCIA, M.C. Decisions under uncertainty and models of agricultural product supply. Notes et Documents Economie et Sociologie Rurales Grignon institut National de la Recherche Agronomique, 1989, 88p.

HALLAM, D., MACHADO, F. Efficiency analysis with panel data: A study of Portuguese dairy farms. European Review of Agricutural Economics. V. 23, p. 79-93, 1996.

HAZEL, P.B.R. e NORTON, R.D. Risk in the farm model. In: Mathematical programming for economic analysis in agriculture, Macmillan, London, p. 76-110, 1986.

HAZEL, P.B.R. A linear alternative to quadratic and semi-variance programing for farm planning under uncertainty. American Journal of Agricultural Economic, v. 53, n. 1, p. 53-62, Feb. 1971.

MARKOWITZ, H. Portifolio seletion. Journal Finance, v 7, n. 1, Mar. 1952.

OGLETHORPE, D.R. The modelling and estimation of farm objective criteria in Northern England: a profit maximisation/MOTAD linear programming analysis. Discussion Paper Department of Agricultural Economics and Food Marketing, Universidade de Newcastle upon Tyne, 1993, 16p.

PARIKH, A. e SHAH, M.K. Measurement of cost inefficiency with safety first of risk. Agricultural Economics, v. 11, n. 2/3, p. 197-296, 1994.

PERES, A.R.P. Baixa produtividade do milho como consequência da tomada de decisão sob condiçōes de risco na agricultura, 1981, Dissertação (Mestrado), ESALQ/USP, $118 \mathrm{p}$.

PERES, F. C. Derived demand for credit under conditions of risk. The Ohio state university, 1976, Tese (Doutorado), 140p.

PERES, F.C. Planejamento da empresa agrícola em condições de risco. In. CONTINI, E.; ARAÚJO, J.D.; OLIVEIRA, A.J. et al. Planejamento da propriedade agrícola, modelos de decisão. Brasília, EMBRAPA - DDT, p. 273-287, 1984.

WANG, J.; WAILES, E.J.; CRAMER, G.L. A shadow-price frontier masurement of efficiency in chinese agriculture. American Journal of Agricultural Economics, v. 78, p. 146-156, Feb, 1996. 